\title{
Astrophysically triggered searches for gravitational waves: status and prospects
}

B Abbott ${ }^{1}$, R Abbott ${ }^{1}$, R Adhikari ${ }^{1}$, P Ajith ${ }^{2}$, B Allen ${ }^{2,3}$, G Allen ${ }^{4}$, R Amin ${ }^{5}$, S B Anderson 1 , W G Anderson ${ }^{3}$, M A Arain ${ }^{6}$, M Araya ${ }^{1}$, H Armandula ${ }^{1}$, P Armor $^{3}$, Y Aso ${ }^{7}$, S Aston $^{8}$, P Aufmuth $^{9}$, C Aulbert $^{2}$, S Babak ${ }^{10}$, S Ballmer ${ }^{1}$, H Bantilan ${ }^{11}$, B C Barish ${ }^{1}$, C Barker ${ }^{12}$, D Barker ${ }^{12}$, B Barr ${ }^{13}$, P Barriga ${ }^{14}$, M A Barton ${ }^{13}$, M Bastarrika ${ }^{13}$, K Bayer $^{15}$, J Betzwieser ${ }^{1}$, P T Beyersdorf ${ }^{16}$, I A Bilenko ${ }^{17}$, G Billingsley ${ }^{1}$, R Biswas ${ }^{3}$, E Black $^{1}$, K Blackburn ${ }^{1}$, L Blackburn ${ }^{15}$, D Blair ${ }^{14}$, B Bland ${ }^{12}$,

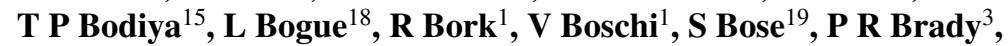
V B Braginsky ${ }^{17}$, J E Brau ${ }^{20}$, M Brinkmann ${ }^{2}$, A Brooks ${ }^{1}$, D A Brown ${ }^{21}$, G Brunet $^{15}$, A Bullington ${ }^{4}$, A Buonanno ${ }^{22}$, O Burmeister ${ }^{2}$, R L Byer ${ }^{4}$, L Cadonati ${ }^{23}$, G Cagnoli ${ }^{13}$, J B Camp ${ }^{24}$, J Cannizzo ${ }^{24}$, K Cannon ${ }^{1}$, $\mathbf{J ~ C a o}^{15}$, L Cardenas ${ }^{1}$, T Casebolt ${ }^{4}$, G Castaldi ${ }^{25}$, C Cepeda ${ }^{1}$, E Chalkley $^{13}$, P Charlton ${ }^{26}$, S Chatterji ${ }^{1}$, S Chelkowski ${ }^{8}$, Y Chen ${ }^{10,27,}$ N Christensen ${ }^{11}$, D Clark ${ }^{4}$, J Clark $^{13}$, T Cokelaer ${ }^{28}$, R Conte $^{29}$, D Cook ${ }^{12}$, T Corbitt $^{15}$, D Coyne ${ }^{1}$, J D E Creighton ${ }^{3}$, A Cumming ${ }^{13}$,

L Cunningham $^{13}$, R M Cutler ${ }^{8}$, J Dalrymple ${ }^{21}$, K Danzmann ${ }^{2,9}$, G Davies $^{28}$, D DeBra ${ }^{4}$, J Degallaix ${ }^{10}$, M Degree ${ }^{4}, \mathbf{V}$ Dergachev $^{30}$, S Desai ${ }^{31}$, R DeSalvo ${ }^{1}$, S Dhurandhar ${ }^{32}, \mathbf{M}$ Díaz $^{33}$, J Dickson ${ }^{34}$, A Dietz $^{28}$, F Donovan ${ }^{15}$, K L Dooley ${ }^{6}$, E E Doomes ${ }^{35}$, R W P Drever ${ }^{36}$, I Duke ${ }^{15}$, J-C Dumas ${ }^{14}$, R J Dupuis ${ }^{1}$, J G Dwyer ${ }^{7}$, C Echols ${ }^{1}$, A Effler ${ }^{12}$, P Ehrens ${ }^{1}$,

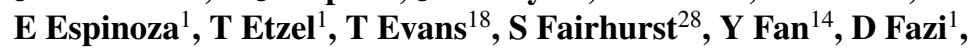
H Fehrmann ${ }^{2}$, M M Fejer ${ }^{4}$, L S Finn ${ }^{31}$, K Flasch ${ }^{3}$, N Fotopoulos ${ }^{3}$,

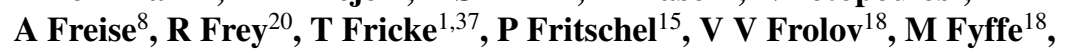
J Garofoli ${ }^{12}$, I Gholami $^{10}$, J A Giaime ${ }^{5,18}$, S Giampanis ${ }^{37}$, K D Giardina ${ }^{18}$, K Goda ${ }^{15}$, E Goetz ${ }^{30}$, L Goggin ${ }^{1}$, G González ${ }^{5}$, S Gossler ${ }^{2}$, R Gouaty ${ }^{5}$,

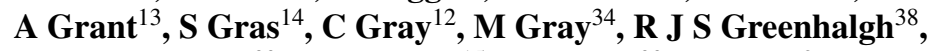

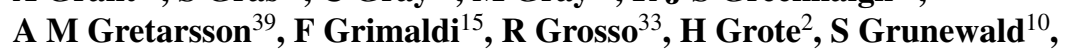
M Guenther ${ }^{12}$, E K Gustafson ${ }^{1}$, R Gustafson ${ }^{30}$, B Hage ${ }^{9}$, J M Hallam ${ }^{8}$, D Hammer ${ }^{3}$, C Hanna ${ }^{5}$, J Hanson ${ }^{18}$, J Harms ${ }^{2}$, G Harry ${ }^{15}$, E Harstad ${ }^{20}$, K Hayama $^{33}$, T Hayler ${ }^{38}$, J Heefner ${ }^{1}$, I S Heng ${ }^{13}$, M Hennessy ${ }^{4}$,

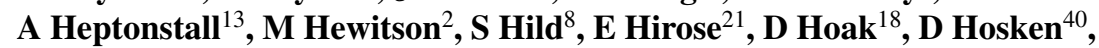

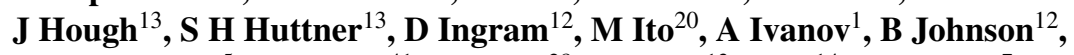
W W Johnson ${ }^{5}$, D I Jones ${ }^{41}$, G Jones ${ }^{28}$, R Jones ${ }^{13}$, L Ju ${ }^{14}$, P Kalmus ${ }^{7}$, V Kalogera ${ }^{42}$, S Kamat ${ }^{7}$, J Kanner ${ }^{22}$, D Kasprzyk ${ }^{8}$, E Katsavounidis ${ }^{15}$, K Kawabe $^{12}$, S Kawamura ${ }^{43}$, F Kawazoe ${ }^{43}$, W Kells ${ }^{1}$, D G Keppel ${ }^{1}$,

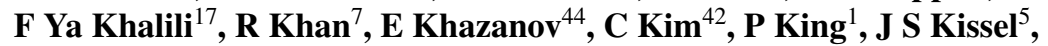
S Klimenko ${ }^{6}$, K Kokeyama ${ }^{43}$, V Kondrashov ${ }^{1}$, R K Kopparapu ${ }^{31}$, D Kozak $^{1}$, I Kozhevatov ${ }^{44}$, B Krishnan ${ }^{10}$, P Kwee ${ }^{9}$, P K Lam ${ }^{34}$, M Landry ${ }^{12}$, M M Lang ${ }^{31}$, B Lantz ${ }^{4}$, A Lazzarini ${ }^{1}$, M Lei ${ }^{1}$, N Leindecker ${ }^{4}$, V Leonhardt ${ }^{43}$, I Leonor ${ }^{20}, K_{\text {Libbrecht }}{ }^{1}$, H Lin $^{6}$, 
P Lindquist $^{1}$, N A Lockerbie ${ }^{45}$, D Lodhia ${ }^{8}$, M Lormand ${ }^{18}, \mathbf{P}$ Lu $^{4}$, M Lubinski ${ }^{12}$, A Lucianetti ${ }^{6}$, H Lück ${ }^{2,9}$, B Machenschalk ${ }^{2}$, M MacInnis $^{15}$,

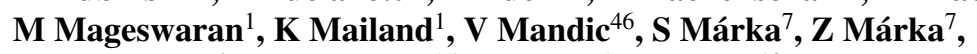

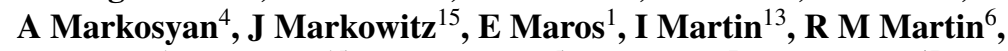
J N Marx ${ }^{1}$, K Mason ${ }^{15}$, F Matichard ${ }^{5}$, L Matone ${ }^{7}$, R Matzner ${ }^{47}$, N Mavalvala ${ }^{15}$, R McCarthy ${ }^{12}$, D E McClelland ${ }^{34}$, S C McGuire ${ }^{35}$, M McHugh $^{48}$, G McIntyre ${ }^{1}$, G McIvor ${ }^{47}$, D McKechan ${ }^{28}$, K McKenzie $^{34}$, T Meier ${ }^{9}$, A Melissinos ${ }^{37}$, G Mendell $^{12}$, R A Mercer ${ }^{6}$, S Meshkov $^{1}$, C J Messenger ${ }^{2}$, D Meyers ${ }^{1}$, J Miller ${ }^{1,13}$, J Minelli ${ }^{31}$, S Mitra ${ }^{32}$, V P Mitrofanov ${ }^{17}$, G Mitselmakher ${ }^{6}$, R Mittleman $^{15}$, O Miyakawa $^{1}$, B Moe $^{3}, \mathbf{S}$ Mohanty ${ }^{33}, \mathbf{G}_{\text {Moreno }}{ }^{12}$, K Mossavi ${ }^{2}$, C MowLowry $^{34}$, G Mueller $^{6}$, S Mukherjee ${ }^{33}$, H Mukhopadhyay ${ }^{32}$, H Müller-Ebhardt ${ }^{2}$,

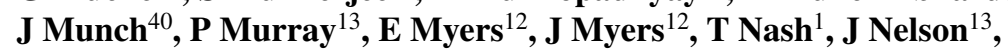
G Newton ${ }^{13}$, A Nishizawa ${ }^{43}, K_{\text {Numata }}{ }^{24}, \mathbf{J}$ O'Dell ${ }^{38}, \mathbf{G}$ Ogin $^{1}$, B O'Reilly ${ }^{18}$, R O'Shaughnessy ${ }^{31}$, D J Ottaway ${ }^{15}$, R S Ottens ${ }^{6}$, H Overmier ${ }^{18}$, B J Owen ${ }^{31}$, Y Pan ${ }^{22}$, C Pankow ${ }^{6}$, M A Papa ${ }^{3,10}$, V Parameshwaraiah ${ }^{12}$, P Patel $^{1}$, M Pedraza $^{1}, \mathbf{S}$ Penn $^{49}$, A Perreca $^{8}$, T Petrie ${ }^{31}$, I M Pinto ${ }^{25}$, M Pitkin ${ }^{13}$, H J Pletsch ${ }^{2}$, M V Plissi ${ }^{13}$, F Postiglione ${ }^{29}$, M Principe ${ }^{25}$, R Prix $^{2}$, V Quetschke ${ }^{6}$, F Raab $^{12}$, D S Rabeling ${ }^{34}$, H Radkins $^{12}$, N Rainer ${ }^{2}$, M Rakhmanov ${ }^{50}$, M Ramsunder ${ }^{31}$, H Rehbein ${ }^{2}$, S Reid ${ }^{13}$, D H Reitze ${ }^{6}$, R Riesen $^{18}$, K Riles $^{30}$, B Rivera ${ }^{12}$, N A Robertson ${ }^{1,13}$, C Robinson ${ }^{28}$, E L Robinson ${ }^{8}$, S Roddy $^{18}$, A Rodriguez ${ }^{5}$, A M Rogan ${ }^{19}$, J Rollins ${ }^{7}$, J D Romano ${ }^{33}$, J Romie $^{18}$, R Route ${ }^{4}$, S Rowan ${ }^{13}$, A Rüdiger ${ }^{2}$, L Ruet ${ }^{15}$, P Russell ${ }^{1}$, K Ryan $^{12}$, S Sakata ${ }^{43}$, M Samidi ${ }^{1}$, L Sancho de la Jordana ${ }^{51}$, V Sandberg ${ }^{12}$, V Sannibale ${ }^{1}, \mathbf{S}$ Saraf ${ }^{52}$, P Sarin ${ }^{15}$, B S Sathyaprakash ${ }^{28}$, S Sato $^{43}$, P R Saulson ${ }^{21}$, R Savage ${ }^{12}$, P Savov ${ }^{27}$, S W Schediwy ${ }^{14}$, R Schilling $^{2}$, R Schnabel ${ }^{2}$, R Schofield ${ }^{20}$, B F Schutz ${ }^{10,28}$, P Schwinberg ${ }^{12}$, S M Scott ${ }^{34}$, A C Searle ${ }^{34}$, B Sears ${ }^{1}$, F Seifert ${ }^{2}$, D Sellers ${ }^{18}$, A S Sengupta ${ }^{1}$, P Shawhan ${ }^{22}$, D H Shoemaker ${ }^{15}$, A Sibley ${ }^{18}$, X Siemens ${ }^{3}$, D Sigg ${ }^{12}$, S Sinha $^{4}$, A M Sintes ${ }^{10,51}$, B J J Slagmolen ${ }^{34}$, J Slutsky ${ }^{5}$, J R Smith ${ }^{21}$, M R Smith ${ }^{1}$, N D Smith ${ }^{15}$, K Somiya ${ }^{2,10}$, B Sorazu ${ }^{13}$, L C Stein ${ }^{15}$, A Stochino ${ }^{1}, \mathbf{R}_{\text {Stone }}^{33}$, K A Strain ${ }^{13}$, D M Strom ${ }^{20}$, A Stuver $^{18}$, T Z Summerscales ${ }^{53}$, K-X Sun ${ }^{4}$, M Sung S $^{5}$ P J Sutton ${ }^{28}$, H Takahashi $^{10}$, D B Tanner ${ }^{6}$, R Taylor ${ }^{1}$, R Taylor ${ }^{13}$, J Thacker ${ }^{18}$, K A Thorne ${ }^{31}$, K S Thorne ${ }^{27}$, A Thüring ${ }^{9}$, K V Tokmakov ${ }^{13}$, C Torres $^{18}$, C Torrie ${ }^{13}$, G Traylor ${ }^{18}$, M Trias ${ }^{51}$, W Tyler ${ }^{1}$, D Ugolini ${ }^{54}$, J Ulmen $^{4}$, K Urbanek $^{4}$, H Vahlbruch ${ }^{9}$, C Van Den Broeck ${ }^{28}$, M van der Sluys ${ }^{42}, S_{\text {Vass }}{ }^{1}$,

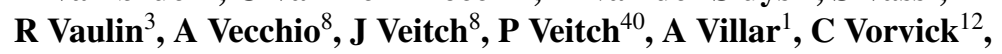

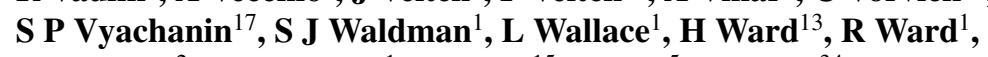
M Weinert ${ }^{2}$, A Weinstein ${ }^{1}, \mathbf{R}$ Weiss ${ }^{15}, \mathbf{S}$ Wen $^{5}$, K Wette $^{34}, \mathbf{J ~ T ~ W h e l a n ~}^{10}$, S E Whitcomb ${ }^{1}$, B F Whiting ${ }^{6}$, C Wilkinson ${ }^{12}$, P A Willems ${ }^{1}$, H R Williams ${ }^{31}$, L Williams ${ }^{6}$, B Willke ${ }^{2,9}$, I Wilmut ${ }^{38}$, W Winkler ${ }^{2}$, C C Wipf ${ }^{15}$, A G Wiseman ${ }^{3}, \mathbf{G}$ Woan $^{13}, \mathbf{R}_{\text {Wooley }}{ }^{18}, \mathbf{J ~ W o r d e n}^{12}, \mathbf{W} \mathbf{W u}^{6}$, I Yakushin $^{18}$, $\mathbf{H}$ Yamamoto $^{1}, \mathbf{Z}_{\text {Yan }}{ }^{14}, \mathbf{S}$ Yoshida $^{50}, \mathbf{M}$ Zanolin $^{39}$, J Zhang $^{30}$, L Zhang ${ }^{1}$, C Zhao ${ }^{14}$, N Zotov ${ }^{55}$, M Zucker ${ }^{15}$, J Zweizig ${ }^{1}$ (The LIGO Scientific Collaboration, http://www.ligo.org) and, F Acernese $e^{56,57}$, M Alshourbagy ${ }^{58,59}, \mathbf{P}$ Amico ${ }^{60,61}$, F Antonucci ${ }^{62}$,

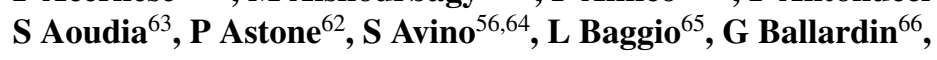


F Barone ${ }^{56,57}$, L Barsotti ${ }^{58,59}$, M Barsuglia ${ }^{67}$, Th S Bauer ${ }^{68}$, S Bigotta $^{58,59}$, S Birindelli ${ }^{58,59}$, M A Bizouard ${ }^{67}$, C Boccara ${ }^{69}$, F Bondu $^{63}$, L Bosi $^{60}$, S Braccini ${ }^{58}$, C Bradaschia ${ }^{58}$, A Brillet ${ }^{63}$, V Brisson ${ }^{67}$, D Buskulic ${ }^{65}$, G Cagnoli $^{70}$, E Calloni ${ }^{56,64}$, E Campagna ${ }^{70,71}$, F Carbognani ${ }^{66}$, F Cavalier $^{67}$, R Cavalieri ${ }^{66}$, G Cella ${ }^{58}$, E Cesarini $^{70,72}$, E Chassande-Mottin ${ }^{63}$, A-C Clapson ${ }^{67}$, F Cleva ${ }^{63}$, E Coccia $^{73,74}$, C Corda $^{58,59}$, A Corsi ${ }^{62}$, F Cottone ${ }^{60,61}$, J-P Coulon ${ }^{63}$, E Cuoco ${ }^{66}$, S D'Antonio ${ }^{73}$, A Dari ${ }^{60,61}$, V Dattilo ${ }^{66}$, M Davier ${ }^{67}$, R De Rosa ${ }^{56,64}$, M Del Prete ${ }^{58,75}$, L Di Fiore ${ }^{56}$, A Di Lieto ${ }^{58,59}$, M Di Paolo Emilio ${ }^{73,76}$,

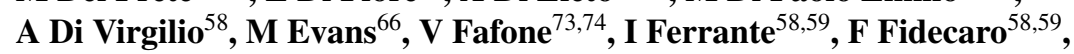
I Fiori $^{66}$, R Flaminio ${ }^{77}$, J-D Fournier ${ }^{63}$, S Frasca ${ }^{62,78}$, F Frasconi ${ }^{58}$, L Gammaitoni ${ }^{60,61}$, F Garufi ${ }^{56,64}$, E Genin ${ }^{66}$, A Gennai ${ }^{58}$, A Giazotto ${ }^{66,58}$,

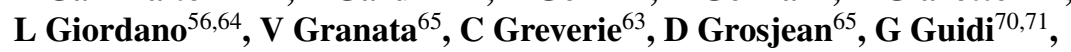

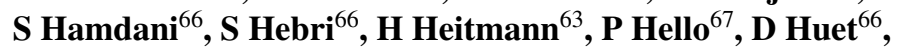

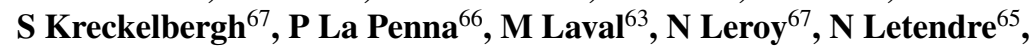
B Lopez ${ }^{66}$, M Lorenzini ${ }^{70,72}$, V Loriette $^{69}$, G Losurdo ${ }^{70}$,

J-M Mackowski ${ }^{77}$, E Majorana ${ }^{62}, \mathbf{C} \mathbf{N}$ Man $^{63}$, M Mantovani $^{59,75}$, F Marchesoni ${ }^{60,61}$, F Marion ${ }^{65}$, J Marque ${ }^{66}$, F Martelli ${ }^{70,71}$, A Masserot ${ }^{65}$, F Menzinger $^{66}$, L Milano ${ }^{56,64}$, Y Minenkov ${ }^{73}, \mathbf{C ~ M o i n s ~}^{66}, \mathbf{J ~ M o r e a u}^{69}$, N Morgado ${ }^{77}, \mathbf{S}$ Mosca $^{56,64}$, B Mours ${ }^{65}$, I Neri ${ }^{60,61}$, F Nocera ${ }^{66}$, G Pagliaroli ${ }^{73}$, C Palomba ${ }^{62}$, F Paoletti ${ }^{66,58}$, S Pardi ${ }^{56,64}$, A Pasqualetti ${ }^{66}$, R Passaquieti ${ }^{58,59}$, D Passuello ${ }^{58}$, F Piergiovanni ${ }^{70,71}$, L Pinard $^{77}$, R Poggiani ${ }^{58,59}$, M Punturo ${ }^{60}$, P Puppo $^{62}$, P Rapagnani $^{62,78}$, T Regimbau $^{63}$, A Remillieux ${ }^{77}$, F Ricci ${ }^{62,78}$, I Ricciardi ${ }^{56,64}$, A Rocchi ${ }^{73}$, L Rolland ${ }^{65}$, R Romano ${ }^{56,57}$, P Ruggi ${ }^{66}$, G Russo ${ }^{56,64}$, S Solimeno ${ }^{56,64}$, A Spallicci ${ }^{63}$, B L Swinkels ${ }^{66}$, M Tarallo ${ }^{58,59}$, R Terenzi ${ }^{73}$, A Toncelli ${ }^{58,59}$, M Tonelli ${ }^{58,59}$, E Tournefier $^{65}$, F Travasso ${ }^{60,61}$, G Vajente $^{79,59}$, J F J van den Brand ${ }^{68,80}$,

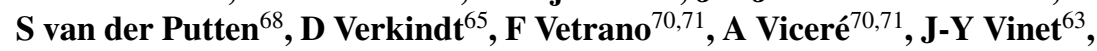
H Vocca ${ }^{60}$ and M Yvert ${ }^{65}$ (Virgo Collaboration, http://www.virgo.infn.it/)

${ }^{1}$ LIGO - California Institute of Technology, Pasadena, CA 91125, USA

${ }^{2}$ Albert-Einstein-Institut, Max-Planck-Institut für Gravitationsphysik, D-30167 Hannover, Germany

${ }^{3}$ University of Wisconsin-Milwaukee, Milwaukee, WI 53201, USA

${ }^{4}$ Stanford University, Stanford, CA 94305, USA

${ }^{5}$ Louisiana State University, Baton Rouge, LA 70803, USA

${ }^{6}$ University of Florida, Gainesville, FL 32611, USA

${ }^{7}$ Columbia University, New York, NY 10027, USA

${ }^{8}$ University of Birmingham, Birmingham, B15 2TT, UK

${ }^{9}$ Leibniz Universität Hannover, D-30167 Hannover, Germany

${ }^{10}$ Albert-Einstein-Institut, Max-Planck-Institut für Gravitationsphysik, D-14476 Golm, Germany

${ }^{11}$ Carleton College, Northfield, MN 55057, USA

12 LIGO Hanford Observatory, Richland, WA 99352, USA

${ }^{13}$ University of Glasgow, Glasgow, G12 8QQ, UK

${ }^{14}$ University of Western Australia, Crawley, WA 6009, Australia

15 LIGO - Massachusetts Institute of Technology, Cambridge, MA 02139, USA

${ }^{16}$ San Jose State University, San Jose, CA 95192, USA

${ }^{17}$ Moscow State University, Moscow, 119992, Russia

${ }^{18}$ LIGO Livingston Observatory, Livingston, LA 70754, USA

${ }^{19}$ Washington State University, Pullman, WA 99164, USA

${ }^{20}$ University of Oregon, Eugene, OR 97403, USA

${ }^{21}$ Syracuse University, Syracuse, NY 13244, USA

22 University of Maryland, College Park, MD 20742, USA

${ }^{23}$ University of Massachusetts, Amherst, MA 01003, USA 
${ }^{24}$ NASA/Goddard Space Flight Center, Greenbelt, MD 20771, USA

${ }^{25}$ University of Sannio at Benevento, I-82100 Benevento, Italy

${ }^{26}$ Charles Sturt University, Wagga Wagga, NSW 2678, Australia

${ }^{27}$ Caltech-CaRT, Pasadena, CA 91125, USA

${ }^{28}$ Cardiff University, Cardiff, CF24 3AA, UK

${ }^{29}$ University of Salerno, 84084 Fisciano (Salerno), Italy

${ }^{30}$ University of Michigan, Ann Arbor, MI 48109, USA

31 The Pennsylvania State University, University Park, PA 16802, USA

${ }^{32}$ Inter-University Centre for Astronomy and Astrophysics, Pune - 411007, India

${ }^{33}$ The University of Texas at Brownsville and Texas Southmost College, Brownsville, TX 78520, USA

${ }^{34}$ Australian National University, Canberra, 0200, Australia

35 Southern University and A\&M College, Baton Rouge, LA 70813, USA

${ }^{36}$ California Institute of Technology, Pasadena, CA 91125, USA

${ }^{37}$ University of Rochester, Rochester, NY 14627, USA

${ }^{38}$ Rutherford Appleton Laboratory, Chilton, Didcot, Oxon, OX11 0QX, UK

${ }^{39}$ Embry-Riddle Aeronautical University, Prescott, AZ 86301, USA

${ }^{40}$ University of Adelaide, Adelaide, SA 5005, Australia

${ }^{41}$ University of Southampton, Southampton, SO17 1BJ, UK

${ }^{42}$ Northwestern University, Evanston, IL 60208, USA

${ }^{43}$ National Astronomical Observatory of Japan, Tokyo 181-8588, Japan

${ }^{44}$ Institute of Applied Physics, Nizhny Novgorod, 603950, Russia

${ }^{45}$ University of Strathclyde, Glasgow, G1 1XQ, UK

${ }^{46}$ University of Minnesota, Minneapolis, MN 55455, USA

47 The University of Texas at Austin, Austin, TX 78712, USA

${ }^{48}$ Loyola University, New Orleans, LA 70118, USA

${ }^{49}$ Hobart and William Smith Colleges, Geneva, NY 14456, USA

50 Southeastern Louisiana University, Hammond, LA 70402, USA

${ }^{51}$ Universitat de les Illes Balears, E-07122 Palma de Mallorca, Spain

52 Sonoma State University, Rohnert Park, CA 94928, USA

53 Andrews University, Berrien Springs, MI 49104, USA

54 Trinity University, San Antonio, TX 78212, USA

${ }^{55}$ Louisiana Tech University, Ruston, LA 71272, USA

56 INFN, Sezione di Napoli, Italy

${ }^{57}$ Università di Salerno, Fisciano (Sa), Italy

${ }^{58}$ INFN, Sezione di Pisa, Italy

${ }^{59}$ Università di Pisa, Pisa, Italy

${ }^{60}$ INFN, Sezione di Perugia, Italy

${ }^{61}$ Università di Perugia, Perugia, Italy

62 INFN, Sezione di Roma, Italy

${ }^{63}$ Departement Artemis - Observatoire de la Côte d'Azur, BP 422906304 Nice, Cedex 4, France

${ }^{64}$ Università di Napoli "Federico II" Complesso Universitario di Monte S.Angelo, Italy

${ }^{65}$ Laboratoire d'Annecy-le-Vieux de Physique des Particules (LAPP), IN2P3/CNRS, Université de Savoie, Annecy-le-Vieux, France

${ }^{66}$ European Gravitational Observatory (EGO), Cascina (Pi), Italy

${ }^{67}$ LAL, Univ Paris-Sud, IN2P3/CNRS, Orsay, France

${ }^{68}$ National Institute for Subatomic Physics, NL-1009 DB Amsterdam, The Netherlands

${ }^{69}$ ESPCI, Paris, France

${ }^{70}$ INFN, Sezione di Firenze, Sesto Fiorentino, Italy

${ }^{71}$ Università degli Studi di Urbino "Carlo Bo", Urbino, Italy

72 Università degli Studi di Firenze, Firenze, Italy

${ }^{73}$ INFN, Sezione di Roma Tor Vergata, Roma, Italy

${ }^{74}$ Università di Roma Tor Vergata, Roma, Italy

${ }^{75}$ Università di Siena, Siena, Italy

${ }^{76}$ Università dell' Aquila, L'Aquila, Italy

77 LMA, Villeurbanne, Lyon, France

${ }^{78}$ Università "La Sapienza", Roma, Italy

${ }^{79}$ Scuola Normale Superiore, Pisa, Italy

${ }^{80}$ Vrije Universiteit, NL-1081 HV Amsterdam, The Netherlands

E-mail: zsuzsa@astro.columbia.edu 
Received 10 January 2008, in final form 25 February 2008

Published 15 May 2008

Online at stacks.iop.org/CQG/25/114051

\begin{abstract}
In gravitational-wave detection, special emphasis is put onto searches that focus on cosmic events detected by other types of astrophysical observatories. The astrophysical triggers, e.g. from $\gamma$-ray and x-ray satellites, optical telescopes and neutrino observatories, provide a trigger time for analyzing gravitationalwave data coincident with the event. In certain cases the expected frequency range, source energetics, directional and progenitor information are also available. Beyond allowing the recognition of gravitational waveforms with amplitudes closer to the noise floor of the detector, these triggered searches should also lead to rich science results even before the onset of Advanced LIGO. In this paper we provide a broad review of LIGO's astrophysically triggered searches and the sources they target.
\end{abstract}

PACS numbers: $04.80 . \mathrm{Nn}$, 95.55.Ym, 95.85.Sz, 97.80.Rz

\title{
1. Introduction
}

Coalescing binaries, supernovae, gamma-ray bursts (GRBs), soft gamma-ray repeaters (SGRs) and other transient sources are not only interesting candidates for gravitational-wave $(\mathrm{GW})$ searches but may also be observed by other means, such as gamma-rays, x-rays, visible light and neutrinos. Therefore GW searches can take advantage of the astrophysical events detected by such independent observatories. Correlation in time (and direction when available) between candidate events in the LIGO/Virgo detectors [1, 2] and the astrophysical trigger event can greatly increase the confidence in the eventual claim of a detection of GWs. Search strategies can be optimized in this respect [3, 4]. Furthermore, several long-term goals of GW astrophysics require detection of astrophysical events in other channels beyond GWs. For example, any association between short hard GRBs and inspiraling neutron star binaries may be confirmed in this manner [5]. The joint detection of GWs and neutrinos together with the observation of the optical light curve from a nearby supernova would greatly enhance our understanding of supernova explosions [6]. The current sensitivity of the LIGO and Virgo detectors allows interesting astrophysical statements to be made by triggered searches for close-by events (see recent results in [7,8]). In this paper we present a brief overview of the strategies employed by the members of the Externally Triggered Searches group of the LIGO Scientific Collaboration (LSC) and Virgo Collaboration and some of the astrophysical sources targeted.

\section{Strategies for externally triggered searches for GWs}

An external trigger provides information about the GW source that allows us to impose additional requirements on candidate signals in the GW data. We can thereby achieve better background rejection and higher sensitivity to real GW signals.

The first requirement imposed during a triggered search for GWs is that the candidate signal be coincident in time (within an astrophysically motivated window) with the external 
trigger. By restricting attention to a subset of the available GW data, a triggered search can be run with a lower event detection threshold than an un-triggered search, giving a higher detection probability at a fixed false alarm probability and better limits in the absence of a detection. Similarly, knowledge of the source direction allows us to search only a relevant part of the sky or, depending on the analysis method, veto candidate events seen in multiple detectors at times not consistent with the expected GW arrival time difference. In some cases electromagnetic observations contain information on the expected GW frequency content (see e.g. [7]), and thus a frequency-band-specific analysis of the GW data set can be performed.

External observations indicate specific progenitor source types for certain trigger events. In such cases model-dependent searches for GWs can be executed. One example is short GRBs, which are thought to be produced by coalescing compact binary systems, and whose GW signal can be detected by matched filtering [8]. Another model-dependent search algorithm is based on van Putten's model for long GRBs [9, 10].

\subsection{Methods for externally triggered searches}

Published LIGO observational results were obtained from cross-correlation analyses of data from multiple detectors $[11,12]$ as well as via a method that uses data from a single detector and finds excess power in astrophysically motivated frequency bands [7].

Coherent network analysis methods, currently under deployment, address the detection and reconstruction of GWs with networks of detectors [13-16]. Based on aperture synthesis, they reconstruct the detector responses to maximize the signal-to-noise ratio of a gravitational wave from a given sky direction. These reconstructed responses are used to construct coherent detection statistics which utilize both the excess power and the cross-correlation energies of the GW signal detected by the network. By combining data from several GW detectors, the coherent methods not only take advantage of the known sky location of a trigger event, but also allow us a consistency test of the events detected in different detectors. Therefore, the coherent methods are expected to have better sensitivity at a given false alarm rate than approaches that test for coincident triggers from individual detector searches. Various coherent statistics, such as the null stream and the network correlation coefficient can be constructed to distinguish a genuine GW signal from the environmental and instrumental artifacts.

Several coherent analysis pipelines are now in use. The coherentWaveBurst pipeline is based on the constraint likelihood method [15] and performs the coherent network analysis in the wavelet domain. The RIDGE [17-19] pipeline uses the Tikhonov regularization scheme [16]. The X-Pipeline is a flexible, general-purpose analysis package [14] for coherent network analysis in the Fourier domain. These pipelines can be used both for the all-sky and triggered searches for gravitational-wave bursts and they provide complementary evaluations of the data.

To study the efficacy of data analysis algorithms for externally triggered searches, realistic Monte Carlo simulations of astrophysically motivated signals are used. The GravEn [20] simulation engine, which has already been used in un-triggered searches during the fourth and fifth LIGO science runs (S4 and S5), is being adapted for use in triggered searches. GravEn simulates the response to gravitational waves of the three LIGO detectors as well as GEO, TAMA and Virgo, and also provides a variety of diagnostic information for each detector site.

\section{GWs from gamma-ray burst engines}

Gamma-ray bursts (GRBs) are intense flashes of $\gamma$-rays which are observed to be isotropically distributed over the sky [21, 22]. The leading hypothesis for most short GRBs (lasting less than 
$\sim 2 \mathrm{~s}$ ) is the merger of neutron star or neutron star-black hole binaries (see [5] and references therein). Long GRBs are associated with hypernovae (see e.g. [23]). In both scenarios the GRB central engine is an accreting solar-mass black hole, and so it is plausible that GRB central engines are also strong emitters of GWs. The GW signal produced by an inspiralling compact binary is well modeled, and can therefore be detected by matched filtering [8, 24]. The GW emission from the binary merger phase and from hypernovae are not well understood, necessitating the use of burst-detection techniques for these sources.

\subsection{Online searches for GWs using GRB triggers}

A near-real time automated analysis was implemented to search LIGO data for GW bursts around triggers received from the IPN/GCN network $[25,26]$. This online search is based on cross-correlating data streams from pairs of detectors. Analysis of LIGO data coincident with 39 GRBs during the second, third and fourth LIGO science runs (S2, S3, S4) found no associated GW burst signals [12]. According to this published LSC result, for S4 the best upper limit on the root-sum-square amplitude of a GW associated with a GRB was $\sim 1 \times 10^{-21} \mathrm{~Hz}^{-1 / 2}$ for circularly polarized waves at $150 \mathrm{~Hz}$.

During S5, November 2005-October 2007, more than 200 GRB triggers were received. For $\sim 70 \%$ of these GRB triggers, at least 2 LIGO detectors were operating, and for $\sim 40 \%$, all three LIGO detectors were collecting data. This large sample of GRBs is the basis for an ongoing search for associated GW burst signals.

\subsection{Joint LIGO-Virgo searches for GWs using GRB triggers}

The advent of data sharing between Virgo and LIGO provides the opportunity to conduct joint searches using high-sensitivity, non-aligned detectors at three distinct locations. Although the Virgo detector is currently somewhat less sensitive than the $4 \mathrm{~km}$ LIGO interferometers, Virgo data can already be beneficial for triggered searches when the Virgo antenna factors are more advantageous at the received trigger position.

We plan to perform a combined analysis of data in coincidence with the $\sim 50$ GRB triggers received during the joint LIGO-Virgo data-taking period (May-Oct. 2007). This analysis will involve one or more of the coherent network methods discussed in section 2.1. In addition, the Virgo Collaboration has independently developed a procedure for GRB triggered searches $[27,28]$, based on the use of the WaveletDetectionFilter (WDF), a wavelet-based transient detection tool $[29,30]$.

While the coherent methods are expected on theoretical grounds to be the most powerful tools for obtaining astrophysically interesting bounds, the coincidence search using WDF will provide robustness against possible noise non-stationarities.

\subsection{GRB population study}

While the GW signals from individual GRBs may be too weak to be detected directly, the small correlations they induce in the data near the GRB trigger time may still be detectable by statistical comparison to data from times not associated with a GRB. The Finn-MohantyRomano algorithm (FMR) [31-33] applies a two-sample test on the sets of inter-detector cross correlations obtained from times with and without GRB triggers. The power of the FMR test increases as $N^{1 / 4}$, where $N$ is the number of triggers at similar redshift, allowing it to accumulate the signal-to-noise ratio over a population of GRBs. This algorithm can place upper limits on the population-averaged energy radiated in GWs [34]. 
We have applied an FMR-inspired algorithm to S2-S3-S4 LIGO data [12]. For the future we plan to incorporate priors using the redshift distribution of observed GRBs from astrophysical literature (see for example [34, 35]), and apply it to the S5 GRB set.

\section{Triggers associated with soft gamma-ray repeaters (SGRs)}

Soft $\gamma$-ray Repeaters (SGRs) are objects (possibly highly magnetized neutron stars [36]) that emit short-duration $\mathrm{x}$ - and $\gamma$-ray bursts at irregular intervals. Occasionally, these objects also emit giant flares lasting hundreds of seconds with peak electromagnetic luminosities reaching $10^{47} \mathrm{erg} \mathrm{s}^{-1}$ [37]. Up to $15 \%$ of short GRBs might be due to SGRs [38]. Since these flares might be accompanied by catastrophic non-radial motion in the stellar matter, galactic SGRs may produce detectable gravitational waves. Furthermore, the X-ray light curve of some SGR giant flares exhibit quasi-periodic oscillations (QPOs) (see e.g. [39]) at well-defined frequencies. These QPOs may be due to seismic modes of the neutron star [39-42] which in turn could emit GWs.

Search methods have been developed which target both the instantaneous gravitational emission at the burst, Flare [43], and the quasi-periodic seismic oscillations of the object following the catastrophic cosmic event [44]. The QPO analysis has been applied to the available LIGO data for the December 27, 2004 hyperflare of SGR1806-20. At the time of the event, the LIGO detectors were under commissioning in preparation for the $\mathrm{S} 4$ science run; only the Hanford $4 \mathrm{~km}$ detector collected data in Astrowatch mode [7, 45] ${ }^{81}$. The best upper limit result by the LSC, for the $92.5 \mathrm{~Hz}$ QPO, corresponds to a GW emission of $7.7 \times 10^{46} \mathrm{erg}$ [7]. This is comparable to the total isotropic energy emitted electromagnetically by the flare, and close to the theoretically expected maximum emission of $\sim 10^{46}$ erg [46-48]. According to a simple isotropic emission model [7], the minimum detectable GW energy released by the source scales with the square of the strain sensitivity. Therefore it is expected, that with over an order of magnitude of sensitivity increase for advanced GW detectors, we will be able to probe the energetics for close-by galactic SGR sources orders of magnitudes below the characteristic $10^{46}$ erg level.

Several hundred SGR bursts were observed electromagnetically during S5. Most of these are attributable to SGR1806-20 and SGR1900+14, both of which have exhibited QPOs in the past [39-42]. These SGR bursts are the target of ongoing searches.

\section{Other sources}

\subsection{Low-mass $\mathrm{x}$-ray binaries}

Low-mass X-ray binaries (LMXBs) are potential sources of GWs [49]. In particular, it has been proposed that $r$-modes inside the neutron star are driven by accretion and generate GWs $[50,51]$. We therefore plan to perform externally triggered searches for GWs from known LMXBs. Investigations of the sensitivity of the global detector network to GW bursts from Sco-X1 are reported in [52]. We also plan to search for coincidences with X-ray data from the Rossi X-Ray Timing Explorer (RXTE) satellite [53]. RXTE can recognize changes in X-ray

${ }^{81}$ Currently the $2 \mathrm{~km}$ interferometer at Hanford (H2), the GEO600 interferometer in Germany and the Virgo detector in Italy remain operable for periods of time and participate in the Astrowatch program (A5). H2 can collect data when its operation does not interfere with Enhanced LIGO commissioning activities, giving us opportunities to continue astrophysical observations at S5 sensitivity levels at a much reduced duty factor. GEO600 is also operational, with a high duty factor, but at a significantly lower sensitivity at low frequencies. Virgo is undergoing a series of upgrades with long commissioning periods, during which data of scientific interest could be collected with much reduced duty factor. 
brightness that occur on a millisecond timescale, thus providing crucial trigger information about $\mathrm{X}$-ray bursts in $\mathrm{LMXBs}^{82}$. In the absence of a detection, upper limits can be used to derive constraints on accretion or the $r$-modes in LXMBs.

\subsection{Pulsar glitches}

Pulsar glitches are observed as step increases in the rotation frequency of pulsars. The increase in rotational energy is $\sim 10^{43} \mathrm{erg}$. These glitches are likely caused by a decoupling between the star's solid crust and superfluid interior (for older pulsars) [55], or by reconfigurations of the crust as spin-down reduces the centrifugal force and the crust reaches breaking strain (for younger pulsars) [56]. In either case, the disruption should excite oscillatory modes throughout the neutron star, leading to the emission of a burst of GWs in the form of a decaying sinusoid, or 'ring-down' [57]. The frequencies and decay times of the modes are determined by the equation of state of the neutron star and its mass and radius; GW observations may be used to constrain these parameters to within a few per cent [58]. The time of the sudden spin-up is limited to within $\sim 2$ min observationally [59], allowing its use as a search trigger.

A Bayesian search method has been developed [60], where the evidence for a ring-down signal is compared with that for a noise model on data around a pulsar glitch trigger. This method includes priors for the signal parameters, as inferred from numerical simulations. The framework also allows the incorporation of alternative signal models which can be used to automatically veto instrumental transients. This method is currently being used to search for GWs associated with pulsar glitches during S5 (including the August 12th, 2006 event of PSR B0833-45 [61]).

\subsection{Neutrinos}

Several astrophysical phenomena, such as core-collapse supernovae, are expected to emit both GWs and neutrinos [6]. The arrival time of neutrinos at detectors, such as Super-Kamiokande [62] and IceCube [63], can therefore serve as triggers for LIGO-Virgo searches. For nearby supernovae, SNEWS [64] will provide an alert and LIGO/Virgo will respond by analyzing the data around the reported event time. Apart from their purely astrophysical interest, GWneutrino coincidences from a supernova would provide new information on neutrino masses [65].

High-energy neutrinos are expected to be emitted along with GWs from a long GRB if the progenitor is a hypernova $[66,67]$ or a compact binary merger [68]. High-energy neutrinos can provide superior directional information in addition to event times. Comparing the source direction reconstructed by neutrino detectors and GW detectors can increase the confidence of a detection. Such an analysis pipeline is under development [69].

\subsection{Optical transients and supernovae}

The current reach of neutrino detectors is limited to our Galactic neighborhood, and thus optical observations are needed to address extragalactic events. According to theoretical calculations, the electromagnetic fluxes expected from plausible sources of GWs should be sufficient to allow the observation of optical counterparts to GW events [70]. Because the light curve of such a source does not appear immediately, an external trigger derived from

${ }^{82}$ RXTE data can enhance our searches for gravitational waves by (a.) providing information on transient events, such as flares from SGRs (b.) providing possible values for parameters of damped normal modes of the neutron star associated with quasi-periodic oscillations [7] and (c.) providing X-ray light curves for low-mass x-ray binaries that can be used as templates in searches for possible gravitational wave signatures in the interferometric data [54]. 
optical observations leads to an uncertainty of several hours in the trigger time, making the data analysis task more challenging but still tractable. Since the sky position is well known, directional analysis methods are applicable. An interesting alternative is to use the source location reconstructed from candidate GW events for follow-up observations with optical telescopes in order to seek confirmation of the event candidate; development of this approach has started during the summer of 2007 [71].

There are other astrophysical transients whose connection to GWs is yet to be explored. For example, blazar flares are powered by accretion onto a supermassive black hole at the center of the host galaxy. Similarly to GRBs, they also have a central engine and jet. Since it has been suggested that some blazars could contain binary black holes [72], they may become future objects of interest for GW searches.

\section{Conclusions}

Interesting results from astrophysically triggered searches using interferometric GW data have been already published [7, 11, 12]. The LIGO detectors have reached their design sensitivities, which already allow us to make specific scientific statements for close-by events, e.g. constraining the source type (or position) of GRB070201, a short-hard GRB event observed to come from a direction overlapping M31 [8].

With the further improvement of interferometric detectors and the application of advanced network methods for externally triggered searches to the LIGO-Virgo network, it is likely that associations between GWs and their electromagnetic counterparts will be confirmed during the lifetime of the advanced detectors.

\section{Acknowledgments}

The authors gratefully acknowledge the support of the United States National Science Foundation for the construction and operation of the LIGO Laboratory, the Science and Technology Facilities Council of the United Kingdom, the Max-Planck-Society, and the State of Niedersachsen/Germany for support of the construction and operation of the GEO600 detector and the Italian Istituto Nazionale di Fisica Nucleare and the French Centre National de la Recherche Scientifique for the construction and operation of the Virgo detector. The authors also gratefully acknowledge the support of the research by these agencies and by the Australian Research Council, the Council of Scientific and Industrial Research of India, the Spanish Ministerio de Educación y Ciencia, the Conselleria d'Economia, Hisenda i Innovació of the Govern de les Illes Balears, the Scottish Funding Council, the Scottish Universities Physics Alliance, The National Aeronautics and Space Administration, the Carnegie Trust, the Leverhulme Trust, the David and Lucile Packard Foundation, the Research Corporation, and the Alfred P. Sloan Foundation. This paper has LIGO Document Number LIGO-P070142$01-\mathrm{Z}$.

\section{References}

[1] Abbott B et al 2004 Nucl. Instrum. Methods Phys. Res. A 517 154-79

[2] Acernese F et al 2006 Class. Quantum Grav. 23635

[3] Arnaud N, Barsuglia M, Bizouard M A, Brisson V, Cavalier F, Davier M, Hello P, Kreckelbergh S and Porter E K 2004 Astropart. Phys. 21 201-21

[4] Mohanty S D, Marka S, Rahkola R, Mukherjee S, Leonor I, Frey R, Cannizzo J and Camp J 2004 Class. Quantum Grav. 21 S765 
[5] Nakar E 2007 Phys. Rep. 442 166-236 (Preprint astro-ph/0701748)

[6] Kotake K, Sato K and Takahashi K 2006 Rep. Prog. Phys. 69 971-1143

[7] Abbott B et al 2007 Phys. Rev. D 76062003

[8] Abbott B et al (Astrophys. J. at press) Preprint 0711.1163

[9] Raffai P, Frei Z, Márka Z and Márka S 2007 Class. Quantum Grav. 24457

[10] van Putten M H, Levinson A, Lee H K, Regimbau T, Punturo M and Harry G M 2004 Phys. Rev. D 69044007

[11] Abbott B et al 2005 Phys. Rev. D 72042002

[12] Abbott B et al 2008 to appear in Phys. Rev. D (Preprint 0709.0766)

[13] Arnaud N, Barsuglia M, Bizouard M A, Brisson V, Cavalier F, Davier M, Hello P, Kreckelbergh S and Porter E K 2003 Phys. Rev. D 68102001 (Preprint gr-qc/0307100)

[14] Chatterji S, Lazzarini A, Stein L, Sutton P, Searle A and Tinto M 2006 Phys. Rev. D 74082005

[15] Klimenko S, Mohanty S, Rakhmanov M and Mitselmakher G 2005 Phys. Rev. D 72122002

[16] Rakhmanov M 2006 Class. Quantum Grav. 23 S673-85 (Preprint gr-qc/0604005)

[17] Hayama K, Mohanty S D, Rakhmanov M and Desai S 2007 Class. Quantum Grav. 24681

[18] Mohanty S D 2002 Class. Quantum Grav. 19 1513-9

[19] Mukherjee S 2003 Class. Quantum Grav. 20925

[20] Stuver A L and Finn L S 2006 Class. Quantum Grav. 23799

[21] Piran T 2005 Rev. Mod. Phys. 76 1143-210 (Preprint astro-ph/0405503)

[22] Mészáros P 2002 Ann. Rev. Astron. Astrophys. 40 137-69 (Preprint astro-ph/0111170)

[23] Stanek K Z et al 2003 Astrophys. J. Lett. 591 L17-20 (Preprint astro-ph/0304173)

[24] Abbott B et al 2008 Phys. Rev. D 77062002 (Preprint 0704.3368)

[25] http://www.ssl.berkeley.edu/ipn3/

[26] http://gcn.gsfc.nasa.gov/

[27] Acernese F et al 2007 Class. Quantum Grav. 24 S671

[28] Corsi A, Cuoco E, Ricci F and Viceré A 2007 Virgo Notes VIR-020A-07

[29] Cuoco E 2005 Virgo Notes VIR-NOT-EGO-1390-308

[30] Cuoco E 2005 Virgo Notes VIR-NOT-EGO-1390-305

[31] Finn L S, Mohanty S D and Romano J D 1999 Phys. Rev. D 60121101

[32] Mohanty S D 2005 Class. Quantum Grav. 22 S1349

[33] Mohanty S D 2006 Class. Quantum Grav. 23 S723

[34] Bromm V and Loeb A 2002 Astrophys. J. 575 111-6 (Preprint astro-ph/0201400)

[35] Guetta D and Piran T 2005 Astron. Astrophys. 435 421-6 (Preprint astro-ph/0407429)

[36] Kouveliotou C et al 1999 Astrophys. J. Lett. 510 L115-8

[37] Woods P M et al 2007 Astrophys. J. 654 470-86 (Preprint astro-ph/0602402)

[38] Nakar E, Gal-Yam A, Piran T and Fox D B 2006 Astrophys. J. 640 849-53

[39] Israel G L et al 2005 Astrophys. J. Lett. 628 L53-6 (Preprint astro-ph/0505255)

[40] Strohmayer T E and Watts A L 2006 Astrophys. J. 653 593-601

[41] Watts A L and Strohmayer T E 2006 Astrophys. J. 637 L117-20

[42] Strohmayer T E and Watts A L 2005 Astrophys. J. 632 L111-4

[43] Kalmus P, Khan R, Matone L and Márka S 2007 Class. Quantum Grav. 24659

[44] Matone L and Márka S 2007 Class. Quantum Grav. 24649

[45] Reitze D 2008 LIGO Document M080011-00-M

[46] de Freitas Pacheco J A 1998 Astron. Astrophys. 336 397-401 (Preprint astro-ph/9805321)

[47] Ioka K 2001 Mon. Not. R. Astron. Soc. 327 639-62 (Preprint astro-ph/0009327)

[48] Horvath J E 2005 Mod. Phys. Lett. A 20 2799-804

[49] Heyl J S 2002 Astrophys. J. 574 L57-L60

[50] Andersson N, Kokkotas K D and Stergioulas N 1999 Astrophys. J. 516 307-314

[51] Bildsten L 1998 Astrophys. J. 501 L89

[52] Hayama K et al 2007 Proceedings of TAUP'07 (Sendai, Japan)

[53] http://heasarc.gsfc.nasa.gov/docs/xte/xtegof.html

[54] Markowitz J 2007 presented at GWDAW12, Conference (13-16 December 2007, Cambridge, MA)

[55] Cheng K S, Pines D, Alpar M A and Shaham J 1988 Astrophys. J. 330 835-46

[56] Franco L M, Link B and Epstein R I 2000 Astrophys. J. 543 987-94 (Preprint astro-ph/9911105)

[57] Thorne K S 1969 Astrophys. J. 1581

[58] Andersson N and Kokkotas K D 1998 Mon. Not. R. Astron. Soc. 299 1059-68 (Preprint gr-qc/9711088)

[59] McCulloch P, Hamilton P, McConnell D and King E 1990 Nature 346

[60] Clark J, Heng I S, Pitkin M and Woan G 2007 Phys. Rev. D 76043003

[61] Flanagan C and Buchner S J 2006 IAU CBAT 595 
[62] Fukuda S (for The Super-KAMIOKANDE Collaboration) 2003 Nucl. Instrum. Meth. Phys. Res. A 501 $418-62$

[63] Ahrens J et al 2004 Astropart. Phys. 20 507-32

[64] Antonioli P et al 2004 New J. Phys. 6114

[65] Arnaud N, Barsuglia M, Bizouard M A, Cavalier F, Davier M, Hello P and Pradier T 2002 Phys. Rev. D 65033010

[66] Waxman E and Bahcall J 1997 Phys. Rev. Lett. 78 2292-5

[67] Vietri M 1998 Phys. Rev. Lett. 80 3690-3

[68] Lee W H and Ramirez-Ruiz E 2007 New J. Phys. 917

[69] Aso Y, Marka Z, Finley C, Dwyer J, Kotake K and Marka S 2008 Class. Quantum Grav. 25114039

[70] Sylvestre J 2003 Phys. Rev. D 68102005

[71] http://geco.phys.columbia.edu/ jap2117/

[72] Hayasaki K, Mineshige S and Sudou H 2007 Publ. Astron. Soc. Japan 59427 